\title{
Industrial Simulation of Metallurgical Logistics
}

\author{
K. Aksyonov, E. Bykov, O. Aksyonova, N. Goncharova, A. Nevolina \\ Department of Information Technology \\ Ural Federal University, Russia
}

\begin{abstract}
The paper focuses on discussion on the method of integration of simulation models used within the enterprise information system. The integration problem is presented on one sample class of models - the real time models that are used in control, diagnostics and decision making processes. The suggested method is based on multi-agent approach with distributed knowledgeable agents. The dynamic model consists in the multi-agent resource conversion process model that supports multi-approach modeling, including discrete-event, agent-based, queuing systems. To substantiate suggested technical decision of the integration module existing message brokers were analyzed. The subject area ontology is presented. It is used for semantic data integration that is required for simulation modeling of technological processes, business processes and logistical processes. In order to achieve a cross-platform system Java language is used for development.
\end{abstract}

Keywords-Simulation; Agent-based modelling; Model integration; Resource conversion processes

\section{INTRODUCTION}

Development of state-of-the-art technologies allows large industrial enterprises to obtain and store vast data volumes that define technological, logistical and commercial problems of an enterprise. These data may be used for simulation modeling of various aspects of its activity. Simulation results may be used for quality control of manufactured products, defect prevention, optimization of logistical and commercial schemes. Integration of these models into the enterprise control circuit by interaction with the corporate information system and development of unified software is a pressing task.

Current ideas of enterprise activity rely on process-based approach. According to it, activity consists of a structured network of processes that consume resources and produce the output. Quality forecasting for the manufactured products, as well as identification of quality degradation incidents, are among the most pressing tasks for metallurgical industry. Simulation modeling is widely used at present for forecasting and optimization of technological, industrial and logistical processes at enterprises of various spheres [1-3]. For this paper we discuss a sample model of metallurgical production for modeling of technological logistics that covers convertor production, hot- and cold-rolled mill products. We are comparing simulation modeling tools from the point of view of this process representation and convenience of model design for the technologist without special programming skills.

Metallurgical manufacture model will be used for analysis of three processes:

Technology of production unit processing, logistical process of production unit load into cars, management process of man-loading during product load.
The model requires definition of the roles of crane and sling operators and their link with the operation of "Loading product unit into the car". Operations need to have corresponding utilized tools set, including various devices, a crane, a train set. The model would show technologist's activity, whose goal is to 1. Direct different order types to corresponding routes, 2. Direct condemned product units for additional treatment. The model will simulate production of pre-defined number of product units (varying from 10 to 10.000).

\section{SYSTEM ARCHITECTURE}

Multi-agent system architecture will further be discussed based on a sample integration system of automated system models of metallurgical production. It contains the following software agents:

Data exchange agent. It is used for actualization of model parameters and data transfer (including experiment results) into corporate information system,

Modeling agent. It is used to solve process control tasks in real time on the basis of real time models,

Message exchange agents. It provides interaction between data exchange and modeling agents. This agent decides when to activate real time model, based on occurring events and activation rules, and also transfers messages into the corporate information system, e.g., into a MES-system or to a corresponding analyst's (specialist, technologist) workstation.

Method of design, development and operation of real time models is based on the methodology of business process analysis and development of information systems. It includes integration of structural and object-oriented approaches, simulation and multi-agent modeling [4] and consists of the following stages:

Design of simulation model in the model definition module,

Running experiments for model verification and adequacy checks in simulation module. BPsim.MAS system is used for this task at the stage of schematic design [4, 5],

Design of real time model for its further use in model integration module and interaction with other sub-systems of the corporate information system. BPsim.SD tool [6] is used for this task at the stage of schematic design.

Use of real time models means that modeling time must be less or equal to a set value, and modeling must be completed before the next portion of data is received from the corporate information system. Thus, the following features need to be considered during the models integration: 
Performance. Architecture of automated system for metallurgical production must be oriented towards maximum use of server resources,

Scalability. Models must be able to run simultaneously on multiple computers, as well as effectively use multi-core and multi-CPU PCs.

To provide these features each model needs to be executed as a standalone process. Special mechanisms, included with the integration module should be used for the interaction of processes.

Integration is suggested to be performed at the data level. Each model performs analysis of data, received from the data storage. Modeling results are transferred either into the data storage or immediately into the corporate information system.

In general case the following data integration levels may be distinguished [7]: physical, logical and semantic. A single ontology of subject area needs to be developed for consideration of semantic properties.

Ontologies are defined as a result on subject area analysis. In our case approach, suggested in [8], has been used. It is based on the Chen's model "entity-relation", since all data is suggested to be stored in relational database. The model has been extended in a way to be able to store other "entityrelation" models and related data.

The method has been extended with such features as availability to process cause-and-effect relations and knowledge of decision making people. Semantic model of the multi-agent resource conversion processes [5] was used for this It was further extended with the elements of logistical projects ontology, presented in [9], and adapted to specific features of logistical problems, related to metallurgical production. Also the ontology included elements of technological and business process. The designed ontology is presented on Figure 1.

Model integration method focuses on several problems [7]. They are briefly discussed further.

\section{MODEL INTERACTION}

The most effective way of interaction of model integration system and automated information system of an enterprise consists in automated obtaining the data required for modeling directly from the automated information system (Figure 2). In order to implement this method we suggest using the Messages queue system, which itself constitutes the architecture and intermediate level software, which collects, stores and distributes messages between subscribers.

Existing message brokers have been analyzed during research. All of them provided guaranteed message delivery between applications.

Since implementation, based on REDIS and Socet.IO message exchange, is simpler, they were selected for data exchange between the automated information system and automated system of metallurgical production.

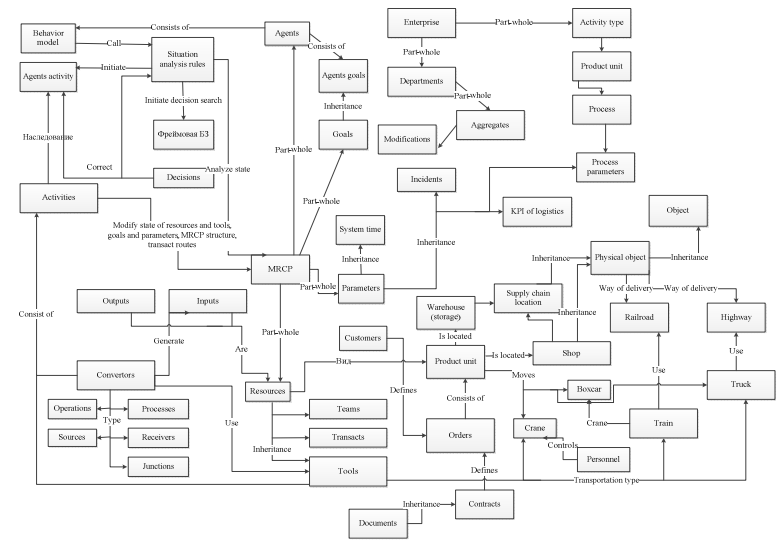

FIGURE I. SUBJECT AREA ONTOLOGY.

\section{DESIGN OF INTEGRATING DATA MODEL}

Integrating data model represents the basis of the common user interface in the integration system. Since the web-interface is suggested for model integration system, a decision, based on JSON and XML standards for the integration model seems reasonable.

The Model-View-Controller or MVC concept is suggested as the main concept of the model integration system development. The concept utilizes several design templates, which allows the application data model, user interface and user interaction are distributed between three specific components, when modification of just one component has minimum impact on other ones. Model integration system includes common classes that implement typical procedures for data obtaining from the automated information system, as well as presentation of modeling results (parameter values, graphs, etc.).

Since the integration module has the multi-agent structure, the agent elements need to correspond to certain elements of MVC. To make things easier, consider a reactive agent with a single rule: "if $a>b$, then $a=a-b$ ". Figure 3 shows the dataflow diagram that presents operation of such agent. Data storages correspond to work memory, which is required to store the variable. Operations on the diagram are all If-Then rules. The agent formulae in software implementation that are stored in "If" and "Then" rules of an agent, are transferred into method definition of the corresponding class. 


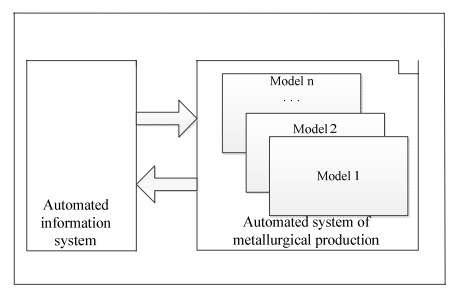

FIGURE II. INTERACTION OF INTEGRATION MODULE WITH THE CORPORATE INFORMATION SYSTEM.

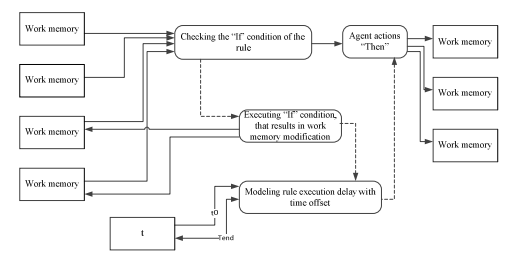

FIGURE III. SAMPLE DFD DIAGRAM FOR THE REACTIVE AGENT WITH ONE RULE.

Thus, from the MVC point of view, work memory represents the Model, while the logical output machine together with agent rules form the Controller. When visualization of modeling results is required by the user, corresponding classes would represent the View.

\section{DEVELOPMENT OF MECHANISMS FOR SEMANTIC INTEGRATION OF DATA SOURCES}

Object-relational mapping is used as a means of data sources semantic integration. This is a programming technology, which allows conversion of incompatible model types between relational data storage and programming objects Such technology is implemented in ORM systems. After analysis two systems have been selected for further development. These are Morphia and Cayenne due to the following factors:

Their features fully satisfy the requirements of model integration system,

Cayenne has a convenient feature of visual development of connection of software classes and entities in the database. This reduces the time required for development and debugging.

The prototype of model integration module for automated information system of metallurgical production has been developed after analysis. Since at this stage, some models of the system are yet to be implemented, testing and running the experiments used the emulated parameter inflow from the technological process. Data required for the simulation model integration module were forwarded to corresponding models for analysis and result output. Model results transfer into the corporate information system for further processing has also been emulated.

\section{SOFTWARE FOR AUTOMATION OF METALLURGICAL PROCESS}

Software for automation of metallurgical production consists of three modules that solve simulation problems.
Process model design module - constructor for development of models by users and analysts,

Enterprise process optimization module implements methods of genetic and multi-agent simulation modeling [4],

Model integration module is used for integration between models and real data sources and use of models for control problems in real time.

Optimization and integration modules may be divided into four modules:

Simulation experiment module that includes model loading from data storage into classes and simulation itself,

Module for saving of results and experiment statistics into data storage,

Experiment design module,

Module for genetic optimization of managed model parameters.

The core of optimization and integration is the simulation experiment module. Input data for the module includes calculated data, acquired with integration module. Output data of the module is further transferred into statistics and optimization modules. Simulation experiment model allows selection of the model, initialization of parameters, and the simulation experiment itself, according to simulation algorithm.

Result saving module accumulates data during the experiment and further saves reports on simulation results into the data storage and spreadsheets.

Experiment design module allows selection of the model and managed parameters, setting the range for managed parameters and generation of full factorial experiment plan. Initial data for selection of managed model parameters and definition of parameter range may be acquired by analysis of data sampling from query constructor module. Query constructor allows factorial analysis of variance and identification of parameters that have a significant influence on the estimated output characteristics. Further these factors should be selected as managed parameters for the process model.

Module for genetic optimization of managed model parameters allows selection of the model and managed parameters as well as an optimization search of such experiment plan (decision population), which would contain an optimal decision (or optimal values of managed model parameters).

\section{CONCLUSION}

Use of simulation modeling for analysis of technological, logistical and business problems of an enterprise is a perspective direction. The discussed method of simulation models integration has been implemented in practice and has successfully passed the tests.

Looking at the convenience of defining an interface for the processes of technological logistics, definition of multi-agent resource conversion process elements, including resources and tools, the modeling module provides the best service. The modeling module requires development in the direction of system integration with the corporate information systems. 


\section{ACKNOWLEDGEMENT}

Research is conducted under the terms of contract № 02.G25.31.0055 (project 2012-218-03-167) under financial support of Ministry of Education and Science of Russian Federation.

\section{REFERENCES}

[1] Aksyonov K. A., Antonova A. S., Kiselyova M. V. 2013. Modeling metallurgical products in AnyLogic and BPsim.MAS, Proceedings of 6th science conference "Simulation. Theory and Practice" IMMOD 2013, volume 2, Kazan, Russia

[2] Karpov Y. G. 2005. Simulation of systems. Introduction to simulation with AnyLogic 5. BHV, St. Petersburg, Russia

[3] Osaba E, Carballedo R and Diaz F. 2012. Simulation tool based on a memetic algorithm to solve a real instance of a dynamic TSP Proceedings of the IASTED International Conference Applied Simulation and Modelling. Napoli, Italy

[4] Aksyonov K.A., Antonova A.S., Spitsina I.A. 2011. Analysis and synthesis of resource conversion processes based on simulation modeling and intelligent agents. // Science and technology news, St. Petersburg State Technical University, № 1 (115). Informatics. Telecommunication. Control. St. Petersburg, p.13-20.

[5] Aksyonov K.A. 2011. Theory and practice of decision support tools Germany, Saarbrucken: LAP LAMBERT Academic Publishing GmbH \& Co. $\mathrm{KG}$

[6] Aksyonov K., Spitsina I., Bykov E., Smoliy E., and Aksyonova O. 2009. Computer-supported software development with BPsim products family - integration of multiple approaches // Proceedings of the 2009 IEEE International Conference on Information and Automation (ICIA). Zhuhai/Macau, China.

[7] Kogalovsky M.R. 2010. Methods of data integration in information systems. Moscow. http://www.cemi.rssi.ru/mei//articles/kogalov1005.pdf

[8] Girardi D, J. Dirnberger, J. Trenkler. 2013. A Meta Model-Based Web Framework for Domain Independent Data Acquisition // ICCGI 2013 The Eighth International Multi-Conference on Computing in the Global Information Technology. Nice, France

[9] Kowalski M., Zelewski S., Bergenrodt D., Klupfel H. 2012. Application of new techniques of artificial intelligence in logistics: an ontologydriven case-based reasoning approach // Proceedings of European Simulation and Modelling Conference 2012. Essen, Germany 